\title{
Risk factors for early hospital readmission after cardiac operations
}

Victor A. Ferraris, MD, PhD,

Suellen P. Ferraris, PhD

R. Christopher Harmon, BS,

Boyd D. Evans, BS

From the Division of Cardiothoracic Surgery, University of Kentucky, Chandler Medical Center, Lexington, Ky, the Department of Cardiovascular Services, Marshall University School of Medicine, Huntington, WVa, and St Mary's Regional Heart Center, Huntington, WVa.

This work was supported in part by an unrestricted grant from the Bayer Corporation, West Haven, Conn.

Read at the Twenty-sixth Annual Meeting of The Western Thoracic Surgical Association, The Big Island, Hawaii, June 21-24, 2000.

Received for publication June 12, 2000; revisions requested Sept 6, 2000; revisions received Dec 12, 2000; accepted for publication Jan 22, 2001.

Address for reprints: Victor A. Ferraris, MD, $\mathrm{PhD}$, Division of Cardiothoracic Surgery, University of Kentucky, Chandler Medical Center, Suite C-208, 800 Rose Street, Lexington, KY 40536-0084 (E-mail: Ferraris@earthlink.net.).

J Thorac Cardiovasc Surg 2001;122:278-86

Copyright (C) 2001 by The American Association for Thoracic Surgery

$0022-5223 / 2001 \$ 35.00+0 \quad \mathbf{1 2 / 6 / 1 1 4 7 7 6}$

doi: $10.1067 / \mathrm{mtc} .2001 .114776$
Objective: Early hospital readmissions after cardiac procedures are both costly and harmful to patients. We investigated the factors that predispose to readmission to develop strategies to minimize this problem.

Methods: As part of a prospective data collection, patients having cardiac procedures at our institution are routinely tracked for 30 days after their discharge from the hospital. We reviewed 2650 patients in our cardiac database who underwent operations over the past 5 years. We used univariate and multivariate statistical techniques to identify risks for readmission.

Results: Of 2574 discharged patients, $252(9.8 \%)$ required readmission. The most common causes of readmission are cardiac $(42 \%)$, pulmonary $(19 \%)$, gastrointestinal $(10 \%)$, extremity complications $(6.7 \%$; deep vein thrombophlebitis, peripheral arterial vascular disease, and saphenous vein harvest site problems), sternal wound problems (7.5\%), and metabolic problems (4\%). Of more than 70 variables studied, only 6 are significant multivariate predictors of readmission: female sex $(P=.002)$; diabetes $(P=.001)$; chronic lung problems $(P=.011)$; increased distance between home and hospital $(P>.001)$; preoperative atrial fibrillation $(P=.002)$; and preoperative chronic renal insufficiency $(P=.002)$. Type of operation, redo procedures, and other intraoperative and postoperative variables are not important multivariate predictors of readmission. Prolonged hospital length of stay for the initial procedure did not cause more frequent readmission. The costs of initial hospitalization (operating room costs combined with postoperative in-hospital costs) were not significantly increased in those patients who required readmission.

Conclusions: The high-risk patient for readmission is a woman with diabetes, chronic lung disease, renal insufficiency, and preoperative atrial fibrillation who lives at a distance from the hospital. Readmission does not depend on periprocedural variables (eg, cardiopulmonary bypass time) or on postoperative complications. High procedural costs from the initial hospitalization do not predispose to readmission. These results suggest interventions that may reduce readmission.

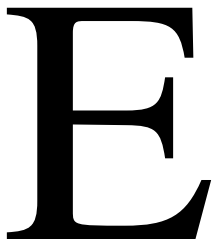

arly, unplanned, cardiac-related hospital readmission following discharge after cardiac operations (READMIT) is common, occurring in $8 \%$ to $24 \%$ of discharged patients. ${ }^{1-4}$ Readmissions that occur within 30 days of discharge are likely related to patient comorbidities and perioperative care. ${ }^{2}$ Additional patient discomfort and expense result from READMIT. ${ }^{5}$ Given the economic importance and the patient-related discomfort associated with READMIT, surprisingly little information is available in the published literature concerning this problem.

We hypothesize that patient-specific and procedure-specific variables might predict READMIT. A corollary of this hypothesis is that interventions may decrease 
the rate of READMIT and improve patient outcomes. This study highlights the risks of READMIT and suggests possible interventions that may limit the occurrence of readmissions.

\section{Methods}

\section{Study Patients}

A total of 2650 patients having cardiac operations at St Mary's Regional Heart Center between October 1994 and September 1999 were included in the study. Of these 2650 patients, 2574 (97.1\%) survived to be discharged. A cardiovascular nurse contacted each of the discharged patients, either by telephone or by direct personal interview, at 30 days after discharge. Thirty-two patients were unable to be contacted at 30 days. For those 32 patients unable to be contacted by the nurse, their surgeon was notified, and contact was made either with a family member or with some other surrogate who had direct personal knowledge of the patient's condition. In this manner each patient who was discharged was accounted for. If a discharged patient was readmitted, either at St Mary's Heart Center or at another hospital, the circumstances of readmission were determined and recorded in the cardiac database. Patients who died outside of the hospital within 30 days after discharge were considered operative deaths for the purpose of this analysis.

\section{Patient Variables and Cardiac Database}

All patients undergoing cardiac procedures at St Mary's Regional Heart Center are included in a cardiac database. Patient variables are stored in the Society of Thoracic Surgeons (STS) database template. This database is available for various quality improvement projects at the St Mary's Regional Heart Center and at the Marshall University Department of Cardiovascular Services. The STS database template contains 556 standard STS patient variables plus 6 additional variables added locally to the database as performance improvement monitors. A full-time, database-dedicated, performance-improvement nurse gathers the data concurrently on each patient. As part of a quality improvement project to limit the number of readmissions after cardiac procedures, the database was used to identify the causes and risk factors for READMIT.

\section{Statistics}

Binary logistic regression analysis was used to model the predictors of READMIT. A commercially available software package was used to perform the calculations (SPSS for Windows, version 10.0.5; SPSS Inc, Chicago, Ill). Seventy-four variables were screened for entry into a logistic regression model. Variables were of 3 types: patient demographics; comorbidities and preoperative risks; and periprocedural variables, including postoperative complications (Appendix 1). Univariate statistical tests of inference were applied to the variables, and this produced a list of candidate variables for entry into the logistic regression model. A variable was considered a candidate predictor variable for the regression model if the univariate tests of inference were significant at less than the .1 probability level and there was no evidence of variable colinearity. ${ }^{6}$ Stepwise logistic regression was used to model predictors of READMIT from the 18 candidate variables identified by means of this process (Appendix 1). Both regression model discrimination and calibration were assessed by using the c-statistic for regression model discrimination and the Hosmer-Lemeshow test statistic for model calibration.

\section{Results \\ Readmissions}

Two hundred fifty-two (9.8\%) of 2574 discharged patients had READMIT. Eight (3.1\%) of 252 patients who were readmitted died during their rehospitalization. Table 1 shows the causes of READMIT. Predictably, cardiac and pulmonary causes for readmission predominate.

\section{Logistic Regression}

Table 2 is a list of 18 candidate variables used in formulating the logistic regression model to identify multivariate determinants of READMIT. Each of the candidate variables exhibits differences between the READMIT and no READMIT groups by means of univariate statistical inference (Table 1 and Appendix 1). When these 18 variables are entered into the stepwise binary logistic regression model, only 6 are significant independent predictors of READMIT (Table 3). Type of operation, redo procedures, and other periprocedural variables are not important predictors of READMIT.

Twenty-one patients were readmitted for treatment of atrial arrhythmias. Only 7 of 21 had atrial arrhythmias before discharge from the initial operation. The development of new postoperative atrial fibrillation is not a risk factor for READMIT, but the existence of preoperative atrial fibrillation is a risk factor. Many of the patients readmitted had preoperative atrial fibrillation but were not necessarily readmitted for control of atrial arrhythmias. Of 22 patients readmitted for congestive heart failure $(\mathrm{CHF}), 10$ had preoperative findings consistent with CHF, but 55 of the 252 readmitted patients had a history of preoperative CHF. This pattern is true for all of the multivariate risk factors for READMIT. The risk factor for READMIT is not necessarily the cause of READMIT, but the risk factor does contribute, in a multivariate way, to READMIT.

The discrimination c-statistic for the final logistic regression analysis is 0.701 , suggesting that about $70 \%$ of the time the model predicts a correct response given a set of positive predictor variables. The Hosmer-Lemeshow test of logistic model calibration suggests that observed probabilities match expected probabilities for READMIT reasonably well. For the purpose of the Hosmer-Lemeshow test, the patients are divided into 10 equal groups, and the predicted probability of READMIT is compared with the observed probability in each of the 10 patient groups. The predicted and observed values of READMIT are compared by means of a $\chi^{2}$ test. The $\chi^{2}$ test result for the Hosmer-Lemeshow test is 5.966 with 8 degrees of freedom, for an overall $\chi^{2}$ signif- 
TABLE 1. Causes of READMIT after cardiac procedures

\begin{tabular}{|c|c|}
\hline Readmission cause ( $\%$ of total patients) & No. of patients \\
\hline Cardiac $(42 \%)$ & 105 \\
\hline Angina & 22 \\
\hline CHF & 22 \\
\hline Rhythm problems & 30 \\
\hline Heart block-bradycardia & 7 \\
\hline Rapid atrial arrhythmias & 21 \\
\hline Vasovagal syncope & 1 \\
\hline Pacemaker exit block & 1 \\
\hline SEMI/AMI & 19 \\
\hline Atypical chest pain & 3 \\
\hline Dressler & 4 \\
\hline Pericardial effusion & 5 \\
\hline Pulmonary (19\%) & 49 \\
\hline Pleural effusion & 31 \\
\hline Pneumonia-bronchitis & 12 \\
\hline COPD exacerbation & 5 \\
\hline Pulmonary embolus & 1 \\
\hline $\mathrm{GI} / \mathrm{GU}(10 \%)$ & 26 \\
\hline UGI bleed-ulcer & 11 \\
\hline SBO & 3 \\
\hline Gastroenteritis-abdominal pain & 3 \\
\hline Perirectal abscess & 1 \\
\hline C. diff. colitis & 1 \\
\hline Pancreatitis & 1 \\
\hline Ischemic bowel & 1 \\
\hline Ruptured appendix & 1 \\
\hline Progression of renal failure & 3 \\
\hline UTI & 1 \\
\hline Sternal wound problems $(7 \%)$ & 18 \\
\hline Deep sternal infection & 13 \\
\hline Sterile sternal dehiscence & 5 \\
\hline Extremity complications (6.7\%) & 17 \\
\hline Saphenous vein harvest site cellulitis-infection & 7 \\
\hline DVT & 9 \\
\hline Thrombosed femoral-popliteal graft & 1 \\
\hline Metabolic disturbances (4\%) & 11 \\
\hline Dehydration & 7 \\
\hline Hyponatremia & 1 \\
\hline Ketoacidosis & 2 \\
\hline Hypoglycemia & 1 \\
\hline Neuropsychiatric $(3.6 \%)$ & 9 \\
\hline Stroke-TIA & 8 \\
\hline Encephalopathy & 1 \\
\hline Anxiety-depression-hallucinations & 4 \\
\hline
\end{tabular}

SEMI, Subendocardial myocardial infarction; $A M I$, acute myocardial infarction; COPD, chronic obstructive pulmonary disease; $U G I$, upper gastrointestinal; $S B O$, small bowel obstruction; $C$. diff., Clostridium difficile; UTI, urinary tract infection; DVT, deep vein thrombophlebitis; TIA, transient ischemic attack.

icance of 0.651. A small $\chi^{2}$ value suggests that there is no significant difference between the observed and expected percentage of READMIT for the final logistic regression model.
On the basis of the characteristics of the logistic analysis, risk factor coefficients can be used to predict the probability of READMIT. The prediction equation is as follows:

$$
\mathrm{P}_{\text {READMIT }}=1 / 1+\mathrm{e}^{\mathrm{X}}
$$

where $\mathrm{P}_{\text {READMIT }}$ is the probability of READMIT and $\mathrm{X}$ is defined as follows:

$$
\begin{gathered}
-0.142+(\text { Sex }+-0.442)+(\text { Diabetes }+-0.448)+(\text { Preatrfib }+-0.648)+ \\
(\text { Chronic obstructive pulmonary disease }+-0.391)+ \\
(\text { Renal }+-0.927)+(\text { ZIPCODE } 1+0.673)+(\text { ZIPCODE } 2+0.481)+ \\
(\text { ZIPCODE } 3+0.293)+(\text { ZIPCODE } 4+-0.324)
\end{gathered}
$$

where Preatrfib is preoperative atrial fibrillation.

The probability of READMIT can be calculated by entering the patient risk factors into a spreadsheet program. The spreadsheet is programmed to calculate the probability of READMIT for patients with various risk factors by using equations 1 and 2. A prototype of this spreadsheet is available from the corresponding author (V.A.F.) on request. Caution should be used in applying this equation because it may be specific for our patients and our geographic setting. In addition, the zip codes for a particular area need to be divided into 5 roughly equal groups, with each successive group representing a greater distance from the hospital.

\section{Resource Use and READMIT}

Neither postoperative complications nor indices of increased resource use (eg, prolonged length of stay and increased hospital cost for the initial hospitalization) were significant predictors of READMIT (Figures 1 and 2).

\section{Discussion}

These results suggest that early readmissions after cardiac procedures are both costly and harmful to patients. More than $3 \%$ of patients who are readmitted die during their rehospitalization. READMIT is at least as lethal an event as is admission for the initial cardiac procedure $(3.1 \%$ vs $2.9 \%$ ). The risk factors that predispose to READMIT include noncardiac comorbidities, such as diabetes, chronic obstructive pulmonary disease, and renal dysfunction. This suggests that other preexisting factors in addition to the initial operation are important risks for readmission. The stress of operation superimposed on these noncardiac comorbidities are important multivariate determinants for READMIT.

The causes of READMIT are heterogeneous. Cardiopulmonary and infectious causes are predominant for READMIT. For example, several patients were readmitted for pleural effusion causing shortness of breath. Multiple causes of pleural effusion range from CHF to retained left pleural fluid from harvest of the left internal thoracic artery. Causes of readmission for pleural effusion may be easily 
TABLE 2. Candidate variables used in the formulation of the logistic regression model to predict READMIT

\begin{tabular}{|c|c|c|c|c|}
\hline Candidate variables & Variable type & $\begin{array}{l}\text { Value for READMIT } \\
\qquad(\mathrm{n}=252)\end{array}$ & $\begin{array}{l}\text { Value for no READMIT } \\
(\mathrm{n}=2322)\end{array}$ & $P$ value \\
\hline Age (y) & Continuous & $64 \pm 11$ & $63 \pm 11$ & .016 \\
\hline Sex (\% women) & Discrete & $39 \%$ & $28 \%$ & .009 \\
\hline Distance from hospital (arbitrary zip code units) & Discrete & 5 categories & & $<.001$ \\
\hline Insurance type (\% Medicare) & Discrete & $59 \%$ & $52 \%$ & .003 \\
\hline Diabetes (\% positive) & Discrete & $69 \%$ & $58 \%$ & .001 \\
\hline Elevated cholesterol & Discrete & $46 \%$ & $42 \%$ & .001 \\
\hline Renal dysfunction & Discrete & $6.7 \%$ & $2.5 \%$ & .001 \\
\hline COPD & Discrete & $28 \%$ & $21 \%$ & .036 \\
\hline Peripheral vascular disease & Discrete & $19 \%$ & $14 \%$ & .100 \\
\hline Preoperative CHF & Discrete & $22 \%$ & $14 \%$ & .004 \\
\hline Preoperative atrial fibrillation & Discrete & $13.9 \%$ & $7.5 \%$ & $<.001$ \\
\hline Preoperative aspirin use & Discrete & $65 \%$ & $71 \%$ & .096 \\
\hline Predicted mortality rate & Continuous & 3.0 & 2.2 & .001 \\
\hline Acute native valve failure & Discrete & $1.6 \%$ & $0.25 \%$ & .002 \\
\hline Intra-aortic balloon required postoperatively & Discrete & $12.3 \%$ & $11 \%$ & .008 \\
\hline Any nonautologous blood products & Discrete & $31 \%$ & $23 \%$ & .010 \\
\hline Deep sternal infection & Discrete & $2 \%$ & $0.08 \%$ & .001 \\
\hline Postoperative delirium & Discrete & $5.6 \%$ & $2.5 \%$ & .038 \\
\hline
\end{tabular}

COPD, Chronic obstructive pulmonary disease.

treatable or may have a poorer prognosis and be difficult to treat. Because of this variability, prediction of READMIT may be difficult. Zitser-Gurevich and colleagues ${ }^{2}$ recognized this difficulty in their study of readmissions within 3 months of coronary artery bypass grafting. These authors suggest that the less serious causes of readmission are easier to prevent. For example, improved support services in the community may decrease the number of readmissions of a less serious nature. These same authors suggest that causespecific readmissions might be a better way to look at the problem of repeat hospitalizations after cardiac procedures. Our study supports this notion. The predictive ability of our regression model for READMIT is fair but could be better (c-statistic $=0.701)$. If patient readmissions for CHF only are subjected to a similar logistic regression model, the predictive ability is better (c-statistic $=0.75$, results not shown). We believe that inclusion of all readmissions is preferable for this type of analysis for 2 reasons. First, as ZitserGurevich and coauthors ${ }^{2}$ point out, the patients with the least serious causes of READMIT may be the easiest ones to treat outside of the hospital with home health services or other measures and hence prevent readmissions. The biggest effect of this study may be on patients with the least serious causes of READMIT. To ignore this segment of the readmission cohort limits the usefulness of this analysis. Second, by looking only at cause-specific readmissions, the sample size of cause-specific readmitted patients becomes an issue. Only 105 patients were readmitted with cardiopulmonary causes of READMIT. Of these 105 patients, 22 had severe CHF. To limit the analysis to this small cohort runs the risk of uncertainties arising in the regression analysis because of sample size and limits the population of study to a much smaller segment of the overall population at risk. For these reasons, we believe that inclusion of all readmissions within 30 days of cardiac operations is a more meaningful way to approach the subject.

The development of postoperative atrial fibrillation is not a risk factor for READMIT, but the existence of preoperative atrial fibrillation is a risk factor. Many of the patients readmitted had preoperative atrial fibrillation and not postoperative atrial fibrillation and were not necessarily readmitted for control of atrial arrhythmias. This means that the existence of preoperative atrial arrhythmias is a marker in patients likely to be readmitted after cardiac procedures but not necessarily the cause of READMIT. All of the multivariate risks for READMIT are chronic comorbidities that differ from the classic risk factors for postoperative mortality. Such factors as prior myocardial infarction, poor ventricular function, hypertension, and redo procedures are not risk factors for READMIT. Preoperative atrial fibrillation fits into the category of a chronic comorbidity that is not a typical risk for mortality after cardiac procedures.

We found that patients who live at a distance from the hospital are at particularly high risk for READMIT. This may be related to the mostly rural population served by St Mary's Regional Heart Center. The population in our study includes a significant portion of the rural mid-Appalachian community. Many of the rural patients are uninsured, elderly, or both. It seems intuitive that these rural patients may have less access to home health services and other outpatient modalities that are potentially helpful in limiting readmissions. From our data, it is not possible to sort out the contributions that rural home 
TABLE 3. Multivariate predictors of READMIT by using logistic regression

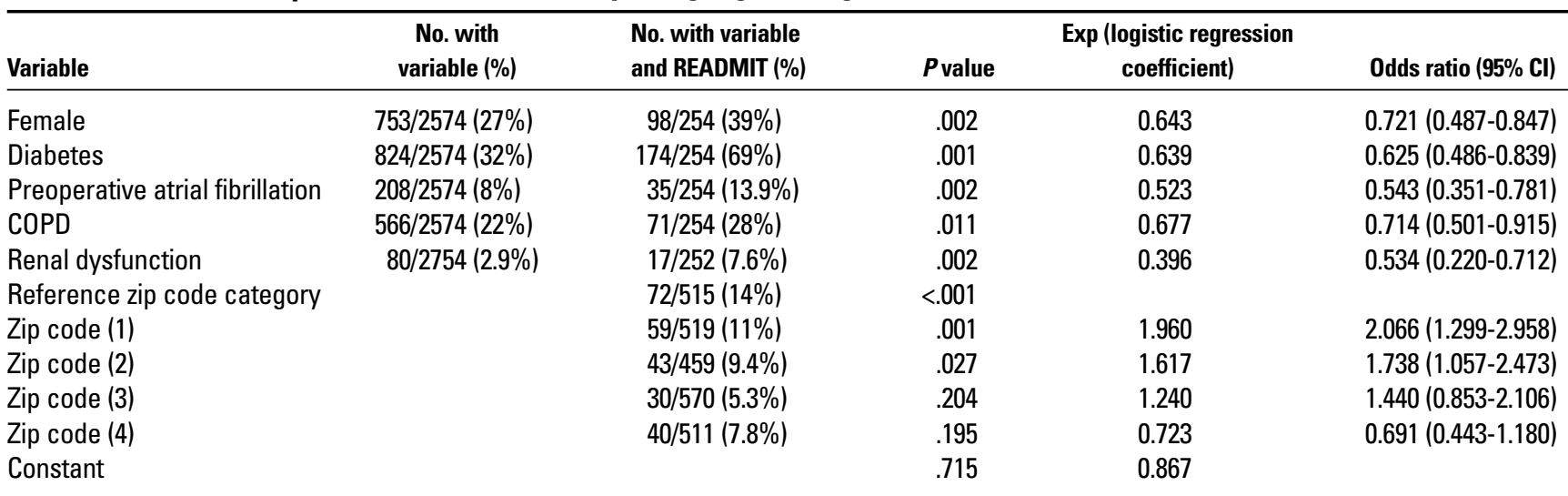

$C O P D$, Chronic obstructive pulmonary disease; $\mathrm{Cl}$, confidence interval.

health care makes to READMIT. READMIT is not more common in patients who live in a rural setting. An index of rural dwelling was assessed by using the US Census data from 1990. The place of residence (urban or rural) does not predict READMIT, but the distance from the hospital does. This suggests that factors such as access to care, including transportation and family support structure, are important contributors to READMIT. On the basis of the census data, rural dwellers did not have a greater risk of READMIT. It is not the rural habitation but the distance from the hospital that predicts READMIT. How these factors interact in the mid-Appalachian region is uncertain.

Our study suggests several interventions that might limit READMIT. Patients with certain comorbidities are at risk for READMIT. We speculate that preoperative treatment of these comorbidities may limit READMIT. Such things as conversion of preoperative atrial fibrillation to sinus rhythm, tight control of diabetes, intensive therapy for chronic lung disease, and adequate hydration to decrease elevated preoperative creatinine levels may help reduce the occurrence of readmission after cardiac operations. There is little or no information available in the published literature to support these suggestions. Most of the published reports about reducing the rate of readmission suggest the use of discharge planning interventions in the postoperative period. ${ }^{4,7-10}$ At present, our study and the intuitive appeal of preoperative intervention to alter comorbidities and limit READMIT are the only supports for this notion. Further controlled trials and quality improvement projects are needed to test this hypothesis.

One might expect that a difficult hospital course associated with the initial cardiac procedure causes more frequent READMIT. Surprisingly, this was not the case. Neither postoperative complications nor indices of increased resource use (eg, prolonged length of stay and increased hospital cost for the initial hospitalization) were significant predictors of READMIT (Figures 1 and 2). Stewart and coworkers ${ }^{11}$ noted that hospital length of stay is not a risk for READMIT. Similarly, Beggs and coauthors ${ }^{3}$ found that prolonged length of stay during the initial hospitalization did not predict readmission after coronary artery bypass grafting. Our results support these observations.

Indices of high resource use do not lead to READMIT. Our previous work suggests that certain patient comorbidities are associated with increased resource use and postoperative morbidity but that events that occur perioperatively are also important predictors of morbidity but not READMIT. ${ }^{12,13}$ There is some duplication between comorbidities associated with increased resource use and those associated with READMIT. Such comorbidities as diabetes, renal dysfunction, and chronic lung disease are common risk factors for both READMIT and increased hospital resource use. This suggests that factors that improve the comorbidity burden of patients before operations will be an effective means of reducing READMIT and limiting costs. This remains an unproven supposition but has intuitive appeal. Thus far, no published studies have taken the next step. What happens to resource use and the rate of readmission if active interventions are done to reduce comorbidity before surgery? The answer to this question is intriguing.

\section{Study Shortcomings}

The logistic regression model discrimination is adequate but not great $(\mathrm{c}$-statistic $=0.701)$. This suggests that other unmeasured variables may be equally important predictors of READMIT. An uncertainty is what happens to patients while they are home after discharge. Many potentially unknown variables may be operative while the patient is recovering at home and may affect READMIT. Such factors as home health care delivery, instructions given to patients before discharge, and other unknown variables may alter READMIT rates. The limitations of the regression model caused by unstudied variables are an 


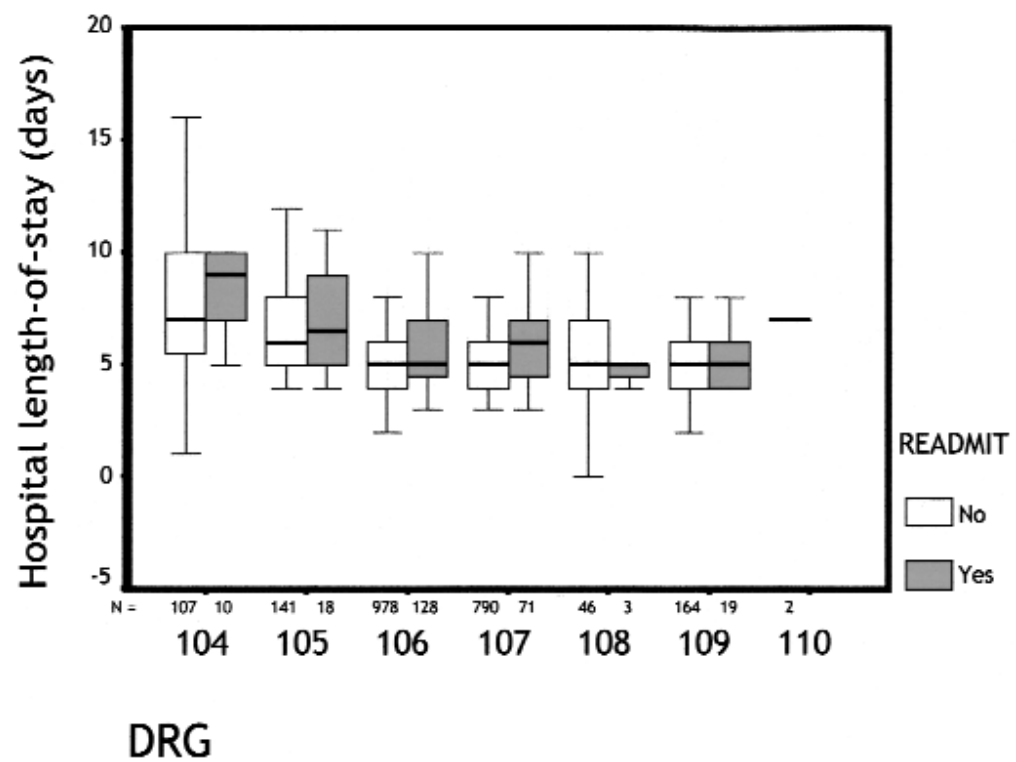

Figure 1. Initial hospital length of stay and READMIT.

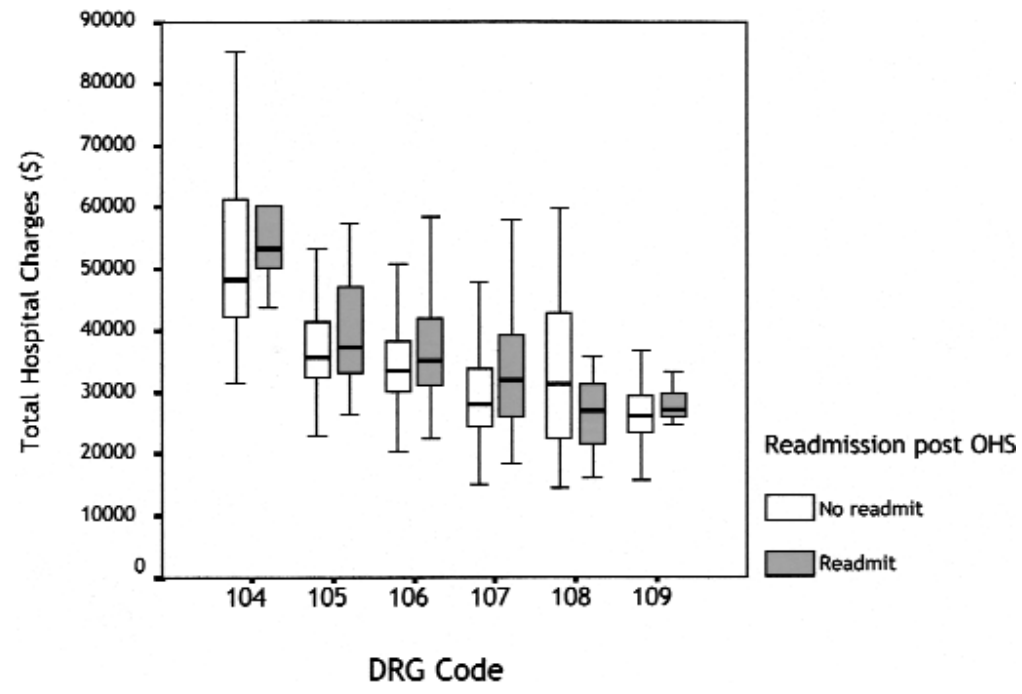

Figure 2. Initial hospital cost and READMIT by diagnosis-related group (DRG) code. OHS, Open heart surgery.

unfortunate fact that cannot be altered because of the database constraints.

We used the zip code of the patient's residence to determine whether the patient lives in a rural setting. This is an oversimplification but may provide useful information. There is evidence to suggest that survival from cardiac events in patients who live in a rural setting, as defined by population density, have worse outcomes. ${ }^{14}$ Rural dwelling did not predict READMIT, but distance from the hospital did. This implies that access to the hospital is an important determinant of READMIT and may be more important than rural living.

\section{Conclusion}

On the basis of multivariate analysis, it is possible to identify a patient at high risk for READMIT. The high-risk patient is a woman with diabetes, preoperative atrial fibrillation, chronic lung disease, and renal insufficiency who lives at a distance from the hospital. READMIT does not depend on periprocedural variables (eg, cardiopulmonary bypass time) or on postoperative complications. High procedural costs from the initial hospitalization do not predispose to READMIT. We speculate that preoperative interventions to minimize or remove patient comorbidities may decrease READMIT and thereby improve outcomes and limit costs. 


\section{References}

1. Sabourin CB, Funk M. Readmission of patients after coronary artery bypass graft surgery. Heart Lung. 1999;28:243-50.

2. Zitser-Gurevich Y, Simchen E, Galai N, Braun D. Prediction of readmissions after CABG using detailed follow-up data: the Israeli CABG Study (ISCAB). Med Care. 1999;37:625-36.

3. Beggs VL, Birkemeyer NJ, Nugent WC, Dacey LJ, O'Connor GT. Factors related to rehospitalization within thirty days of discharge after coronary artery bypass grafting. Best Pract Benchmarking Healthcare. 1996;1:180-6.

4. Stanton BA, Jenkins CD, Goldstein RL, Vander Salm TJ, Klein MD, Aucoin RA. Hospital readmissions among survivors six months after myocardial revascularization. JAMA. 1985;253:3568-73.

5. Ades PA, Huang D, Weaver SO. Cardiac rehabilitation participation predicts lower rehospitalization costs. Am Heart J. 1992;123:916-21.

6. Glantz SA, Slinker BK. Regression diagnostics for logistic regression. In: Glantz SA, Slinker BK, editors. Primer of applied regression and analysis of variance. New York: McGraw-Hill, Inc; 1990. p. 529-68.

7. Lahey SJ, Campos CT, Jennings B, Pawlow P, Stokes T, Levitsky S. Hospital readmission after cardiac surgery. Does "fast track" cardiac surgery result in cost saving or cost shifting? Circulation. 1998;98(Suppl 19):II-35-40.

8. Sternlieb JJ. Exploring the risk of early dismissal following cardiac operations. J Cardiovasc Surg (Torino). 1987;28:120-3.

9. Naylor MD, McCauley KM. The effects of a discharge planning and home follow-up intervention on elders hospitalized with common medical and surgical cardiac conditions. J Cardiovasc Nurs. 1999; $14: 44-54$.

10. D'Agostino RS, Jacobson J, Clarkson M, Svensson LG, Williamson C, Shahian DM. Readmission after cardiac operations: prevalence, patterns, and predisposing factors. J Thorac Cardiovasc Surg. 1999;118:823-32.

11. Stewart RD, Campos CT, Jennings B, Lollis SS, Levitsky S, Lahey SJ. Predictors of 30-day hospital readmission after coronary artery bypass. Ann Thorac Surg. 2000;70:169-74.

12. Ferraris VA, Ferraris SP, Singh A. Operative outcome and hospital cost. J Thorac Cardiovasc Surg. 1998;115:593-603.

13. Ferraris VA, Ferraris SP. Risk factors for postoperative morbidity. $J$ Thorac Cardiovasc Surg. 1996;111:731-41.

14. Stapczynski JS, Svenson JE, Stone CK. Population density, automated external defibrillator use, and survival in rural cardiac arrest. Acad Emerg Med. 1997;4:552-8.

\section{Discussion}

Dr Kevin Linkus (Reno, Nev). When Dr Gundry contacted me to invite my review and discussion of the article, I was very eager and excited to proceed with the task. However, after Dr Ferraris supplied me with the actual text of the article, I was quite concerned regarding my upcoming task. On reading this excellently written article, I realized my college math had long abandoned my knowledge base.

The multivariate analysis of READMIT factors and the application of the regression model have been eloquently performed in this article. The information is extensive and accurately presented. The conclusion about the readmission rate for female sex, diabetes, atrial fibrillation, chronic lung disease, and renal disease is not particularly surprising. Certainly these factors cannot be disputed by any high-volume cardiac surgeon. I was surprised that other factors do not compete with the above, such as severe postoperative complications that would or could lead to possible disability. I was particularly impressed by the $2.9 \%$ mortality rate with 2574 patients discharged of a total of 2650 over a 5-year period. The READMIT rate of $9.8 \%$ is commendable, especially considering the rural practice of Marshall University Medical Center. Follow-up was certainly exhaustive, especially considering the number of patients interviewed. I was impressed at the extensive secondary sources that were contacted to complete the study of those 32 patients who could not be initially interviewed.

I have 3 short questions or comments regarding the article. Our practice in Reno, Nevada, is very similar to yours in West Virginia. We have an extensive population base that is spread out over a very large geographic area. We have 14 outreach clinics covering a wide range requiring a jet aircraft for us to service our 14 clinics. A brief review of our statistics indicates that approximately 1100 cardiac operations were done per year in 1998 and 1999. I noticed that we had an 11\% READMIT rate in 1998 and a 6\% READMIT rate in 1999. Eighteen months ago, we began an extensive home health program in which registered nurses visit our postoperative patients 3 to 14 times per week for each and every patient discharged from our service. You briefly mentioned home health efforts in reducing readmissions to the West Virginia center. What effect would a more involved followup care outreach program have on your results?

Dr Ferraris. I do not know, but certainly we suspect that closer observation in the outpatient setting might reduce readmissions. Viewed another way, it might increase them as well. A lot of times, the home health people detect problems and advise us to examine a patient. In a rural setting that means that the patient comes to the hospital, and often that means readmission. Honestly, I am not sure what the effect of home health or supervised care outside the hospital would be. I think it is something that should be tested.

Dr Linkus. You follow readmissions for only 30 days on the basis of the STS data requirement. We have begun to monitor our patients on a 90-day or 3-month follow-up interval because this is a requirement for most of our private insurers for global fee payment and Medicare statistics. Why did you choose a 30-day follow-up versus a 90-day follow-up?

Dr Ferraris. Thirty-day follow-up was used for 2 reasons. First, it is an STS increment on the database, and we wanted to adhere to the STS format as closely as possible. Second, we believed that readmissions for a cardiac problem or some problem related to an operation were likely to occur within 30 days. Extending the follow-up to 90 days may encompass comorbidities and may not be related to the operation.

Dr Linkus. Finally, in your conclusion you stated that prolonged hospital stay for the initial procedure did not cause more frequent READMIT. We in Nevada, like you, tend to keep our patients longer in the hospital, especially if they live further away from our medical center. Did your study address the inverse of your statement? Did you find that prolonged hospital length of stay reduced readmission and the cost of that event?

Dr Ferraris. That is an interesting question. I do not think I can answer that on the basis of information I have. There is clearly a bias, though. If we know the patient lives far from the hospital, we are willing to have him or her stay in the hospital a little bit longer. Another issue is that we have a rehabilitation center that is attached to the hospital. Many patients who are elderly, who do not have a spouse at home, and who live at a distance spend a week or two in that facility. It is a complex issue, and I just do not have an answer. 
APPENDIX 1. List used to obtain candidate variables for the logistic regression model

\begin{tabular}{|c|c|c|}
\hline Variable* & Type & $\begin{array}{c}P \text { value for statistical } \\
\text { test of inferencet }\end{array}$ \\
\hline Age & Demographic & .016 \\
\hline Sex & Demographic & .001 \\
\hline Ethnicity & Demographic & .613 \\
\hline Height (cm) & Demographic & .325 \\
\hline Weight $(\mathrm{kg})$ & Demographic & .652 \\
\hline Distance from the hospital (based on zip code) & Demographic & $<.001$ \\
\hline $\begin{array}{l}\text { Percentage rural population within zip code of residence } \\
\text { (US Census 1990) }\end{array}$ & Demographic & .110 \\
\hline Insurance type & Demographic & .003 \\
\hline Current cigarette use & Comorbidities or preoperative risks & .645 \\
\hline Length of stay (operating room to discharge) & Comorbidities and preoperative risks & .192 \\
\hline Diabetes & Comorbidities and preoperative risks & .001 \\
\hline Morbid obesity & Comorbidities and preoperative risks & .256 \\
\hline Hypercholesterolemia & Comorbidities and preoperative risks & .001 \\
\hline Renal dysfunction (creatinine >2.0) & Comorbidities and preoperative risks & .001 \\
\hline Hypertension & Comorbidities and preoperative risks & .168 \\
\hline Prior stroke & Comorbidities and preoperative risks & 619 \\
\hline Endocarditis & Comorbidities and preoperative risks & 647 \\
\hline Chronic obstructive lung disease & Comorbidities and preoperative risks & .036 \\
\hline Immune suppression & Comorbidities and preoperative risks & .417 \\
\hline Peripheral vascular disease & Comorbidities and preoperative risks & 100 \\
\hline Carotid arterial disease & Comorbidities and preoperative risks & .921 \\
\hline Prior cardiac procedure & Comorbidities and preoperative risks & .782 \\
\hline Recent myocardial infarction & Comorbidities and preoperative risks & .838 \\
\hline Preoperative congestive heart failure & Comorbidities and preoperative risks & .004 \\
\hline Cardiogenic shock & Comorbidities and preoperative risks & .139 \\
\hline Cardiopulmonary resuscitation in progress & Comorbidities and preoperative risks & .155 \\
\hline Preoperative atrial fibrillation & Comorbidities and preoperative risks & $<.001$ \\
\hline Inotropes & Comorbidities and preoperative risks & .522 \\
\hline Antiplatelet drugs & Comorbidities and preoperative risks & .122 \\
\hline Aspirin & Comorbidities and preoperative risks & .096 \\
\hline Heparin & Comorbidities and preoperative risks & .843 \\
\hline Thrombolysis & Comorbidities and preoperative risks & .643 \\
\hline Prior PTCA & Comorbidities and preoperative risks & .552 \\
\hline Ejection fraction & Comorbidities and preoperative risks & .822 \\
\hline Crossclamp time & Periprocedural variables & .655 \\
\hline Pump time & Periprocedural variables & .165 \\
\hline Predicted mortality & Periprocedural variables & $<.001$ \\
\hline Type of operation & Periprocedural variables & .714 \\
\hline
\end{tabular}


APPENDIX 1. Cont'd

\begin{tabular}{|c|c|c|}
\hline Variable* & Type & $\begin{array}{c}P \text { value for statistical } \\
\text { test of inferencet }\end{array}$ \\
\hline Diagnosis-related group code & Periprocedural variables & .193 \\
\hline Hemodynamic instability & Periprocedural variables & .269 \\
\hline Evolving acute myocardial infarction & Periprocedural variables & .321 \\
\hline Mechanical valve dysfunction & Periprocedural variables & .257 \\
\hline Catheterization laboratory crash & Periprocedural variables & .231 \\
\hline Acute native valve failure & Periprocedural variables & .002 \\
\hline Unstable angina (intravenous nitroglycerin) & Periprocedural variables & .268 \\
\hline Left main obstruction $>50 \%$ & Periprocedural variables & .367 \\
\hline Aortic dissection & Periprocedural variables & .489 \\
\hline Intra-aortic balloon postoperatively & Periprocedural variables & .008 \\
\hline Any nonautologous blood products & Periprocedural variables & .010 \\
\hline Any reoperation in the postoperative period & Periprocedural variables & .956 \\
\hline Perioperative myocardial infarction & Periprocedural variables & .580 \\
\hline Any postoperative infection & Periprocedural variables & .125 \\
\hline Deep sternal infection & Periprocedural variables & $<.001$ \\
\hline Septicemia & Periprocedural variables & .892 \\
\hline Urinary tract infection & Periprocedural variables & .645 \\
\hline Leg infection & Periprocedural variables & .590 \\
\hline Postoperative stroke & Periprocedural variables & .937 \\
\hline Postoperative TIA & Periprocedural variables & .788 \\
\hline Delirium & Periprocedural variables & .038 \\
\hline Coma & Periprocedural variables & .788 \\
\hline Prolonged ventilator & Periprocedural variables & .122 \\
\hline Pulmonary embolism & Periprocedural variables & .810 \\
\hline Postoperative pulmonary edema & Periprocedural variables & .311 \\
\hline ARDS & Periprocedural variables & .890 \\
\hline Postoperative pneumonia & Periprocedural variables & .156 \\
\hline Any postoperative vascular complication & Periprocedural variables & .925 \\
\hline Postoperative valvular dysfunction & Periprocedural variables & .810 \\
\hline Postoperative renal failure & Periprocedural variables & .448 \\
\hline Postoperative heart block & Periprocedural variables & .920 \\
\hline Gastrointestinal complications & Periprocedural variables & .276 \\
\hline Postoperative atrial fibrillation & Periprocedural variables & .134 \\
\hline Postoperative cardiac tamponade & Periprocedural variables & .889 \\
\hline Multiorgan failure & Periprocedural variables & .663 \\
\hline Postoperative cardiac arrest & Periprocedural variables & .523 \\
\hline
\end{tabular}

PTCA, Percutaneous transluminal coronary angioplasty; TIA, transient ischemic attack; ARDS, adult respiratory distress syndrome.

*Variables in bold are candidate variables that were entered into the logistic regression model.

tAn unpaired $t$ test was used for continuous variables, and the Fisher exact test was used for discrete variables. 